\section{EL VÍDEO DIDÁCTICO COMO SOPORTE PARA UN b-LEARNING SOSTENIBLE}

\author{
Miguel Ángel del Casar Tenorio \\ Rafael Herradón Díez \\ Departamento IAC, EUIT Telecomunicación-UPM, \\ Ctra. Valencia Km. 7, Campus Sur \\ miguelangel.delcasar@upm.es \\ rafael.herradon@upm.es
}

\begin{abstract}
This paper deals about one innovative educative experience carried out with pre-graduated students of the Sistemas de Telecomunicación title in the EUIT Telecomunicación-Technical University of Madrid in which a blended learning methodology, based on the mixture of a face-to-face in-classroom conventional approach blended with some tools derived from the Information and Communications Technology, has been employed in order to foster the active participation and implication of the students in their own educative process. More specifically a good deal of audio-visual material have been elaborated ad hoc and offered to the students by means of the Internet platform in order to make easy and improve their hands-on laboratory skills.
\end{abstract}

KEY WORDS: Educative videos, b-learning, ICT technologies, reusable learning objects, educative sustanaibility, collaborative working.

\section{INTRODUCCIÓN}

Hoy día resulta incontrovertible el profundo impacto que las tecnologías de la información y la comunicación (TICs) tienen en el quehacer diario en nuestra vida (al menos para los habitantes de las sociedades denominadas del primer mundo). El campo de la educación, y más específicamente el universitario, no ha quedado al margen de las enormes posibilidades que ofrecen las TIC y así se han ido incorporando paulatinamente en la labor de docentes y alumnos, substituyendo o ampliando las herramientas pedagógicas convencionales con diferentes manifestaciones: pizarra electrónica, presentaciones power point en lugar de transparencias sobre acetato, multi-plataformas web para realización de exámenes en lugar de papel, libros electrónicos, laboratorios remotos, etc. La integración, y consiguiente sinergía, del abanico de recursos pedagógicos sobre soporte electrónico (e-learning) junto con las me-

\section{$A$ b-LEARNING SUSTAINABLE APPROACH BY MEANS OF DIDACTIC VIDEOS}

RESUMEN: En este artículo se describe una experiencia de innovación educativa realizada en el marco de la titulación de Sistemas de Telecomunicación de la EUITT de la Universidad Politécnica de Madrid, en la que se ha intentado graduar la dificultad de acceso de los alumnos al uso de ciertos laboratorios altamente tecnificados, por medio de instrumentos pedagógicos basados en el uso de tecnologías de la información y las comunicaciones. Se presenta por tanto, un escenario propicio para la aplicación de una metodología educativa de formación combinada o blended-learning que potencie el proceso de aprendizaje de los estudiantes así como la optimización del uso de los costosos recursos materiales puestos a su disposición.

PALABRAS CLAVE: Videos educativos, b-learning, tecnologias TIC, objetos de aprendizaje reutilizables, sostenibilidad educativa, trabajo colaborativo.

todologías presenciales de clases cara a cara en un aula o laboratorio, constituyen, como muy bien señala Ynoue, 2010, los elementos que definen un proceso docente blended learning (o b-learning).

\section{EL PARADIGMA DEL $\boldsymbol{b}$-LEARNING}

La asociación de diferentes tecnologías, metodologías y actividades que se combinan en un escenario de b-learning, juegan un papel fundamental en la adopción de una estrategia educativa flexible, eficaz y sostenible en el entorno de una educación técnica universitaria de calidad. Cada una de las tres cualidades mencionadas no es sino el fruto obtenido de aprovechar las fortalezas de cada uno de los dos mundos que se concitan, a la vez que se minimizan sus debilidades inherentes. En un amplio estudio sobre el tema 
(Bersin, 2004), nos hace notar las características distintivas de la educación puramente presencial y de la denominada e-learning. El diagrama de la figura 1 ilustra esquemáticamente cómo el paradigma semi-presencial consustancial al b-learning viene a nutrirse de las ventajas asociadas a ambos mundos educativos (aparentemente disjuntos), tratando de soslayar sus respectivas debilidades.

Los teóricos del diseño pedagógico como Dunn y Griggs, 2000, indican que un elevado porcentaje de las personas

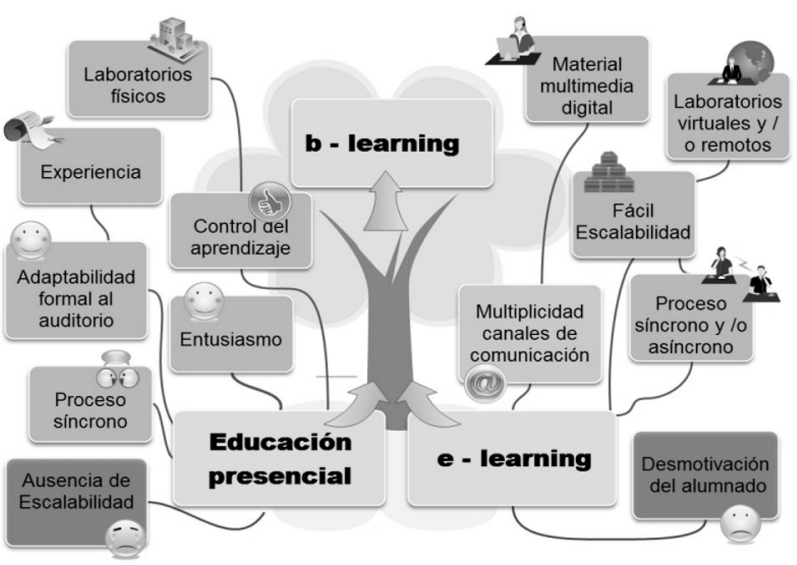

Figura 1. Genealogía de la estrategia b-learning.

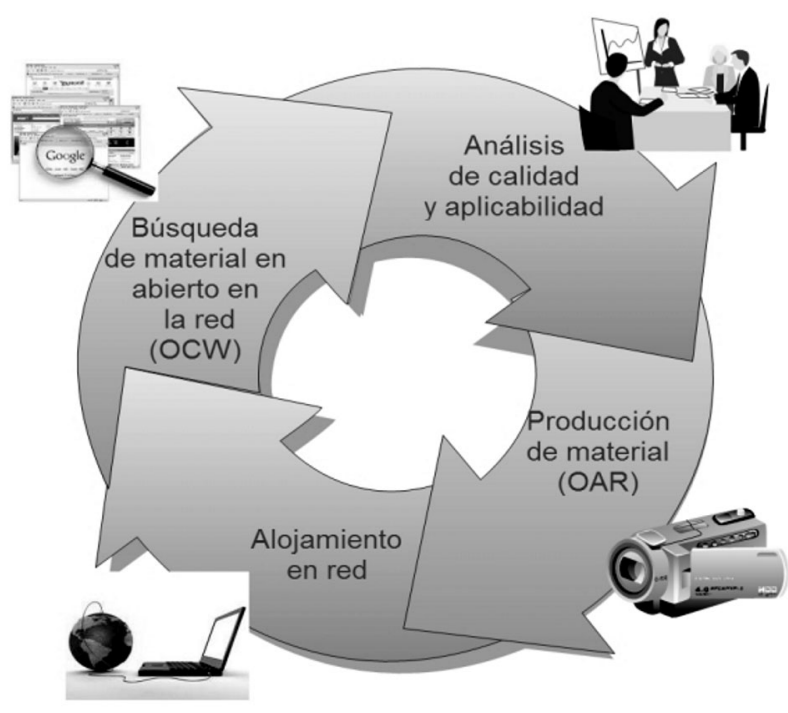

Figura 2. Ciclo de trabajo de búsqueda-generación de OAR. adquieren conocimientos a través de los estilos de aprendizaje visual y auditivo, siendo mucho menor el grupo de individuos que prefieren la modalidad kinestésica. A la luz de estas conlusiones parece claro que un buen diseño de cualquier sistema educativo, y en particular de uno con estrategia b-learning, deberá aportar una buena dosis de contenido audiovisual a su auditorio potencial. Ocurre, sin embargo, la singular paradoja de que en un mundo como el actual completamente dominado precisamente por la tecnología audiovisual, sea uno de sus hijos más preeminentes, como es el vídeo, uno de los recursos educativos menos explotado en el ámbito de la educación universitaria.

\section{El VÍdeO COMO heRRAMIENTA EDUCATIVA}

Desde sus más primigenios orígenes el vídeo ha sido reconocido como un posible instrumento pedagógico de primera magnitud en las enseñanzas técnicas. En palabras de Gruber "el uso del vídeo en clase facilita la construcción de un conocimiento significativo dado que se aprovecha el potencial comunicativo de las imágenes, los sonidos y las palabras para transmitir una serie de experiencias que estimulen los sentidos y los diferentes estilos de aprendizaje en los alumnos. Esto permite concebir una imagen más real de un concepto".

En el contexto actual sobreentendemos vídeo sobre soporte digital, ya sea nativo o digitalización de un material videográfico analógico, y que por su propia naturaleza permite adicionalmente las ventajas de:

- Facilidad de acceso y distribución. Ya sea sobre soporte físico (CD, DVD, memorias flash-drive...) o a través de Internet (plataforma YouTube, repositorios y otros).

- Multiplicidad de plataformas de reproducción tanto en terminales fijos (TV, ordenador personal de sobremesa) como portátiles (ordenador portátil, notebook, smartphone, disco duro multimedia, tablet $P C$, vídeo consola, reproductor MP4, etc.).

- Facilidad de generación y edición. A partir de diferentes materiales fuente (fotografías, textos, películas, animaciones, música, voz, etc.) y sin necesidad de utilizar recursos muy costosos (simplemente un ordenador personal y el software de edición no-lineal de vídeo adecuado) se puede generar un material pedagógico 
de excelente calidad que pueda ser re-editado ulteriormente para acomodarlo a diferentes escenarios de utilización.

Sentadas las premisas que justifican el uso del vídeo como herramienta pedagógica propicia al estilo de aprendizaje de un porcentaje notablemente alto de la población y habiendo puesto en valor sus caracteristicas técnicas ideales para una estrategia de $b$-learning sostenible (el mismo material puede utilizarse y difundirse entre una infinidad de alumnos sin incremento alguno de coste), cabe preguntarse por las características técnicas y de contenido más adecuadas para que ejerza la labor pedagógica pretendida de la manera más eficaz posible. La respuesta a ambas cuestiones las encontraremos a raíz de la siguiente discusión.

\section{El VÍdEO DISEÑAdO COMO OAR}

Desde su introducción hacia 1992 por Wayne Hodgins, el concepto de Objeto de Aprendizaje Reutilizable ${ }^{1}$ (OAR) ha sido eje de numerosa atención y debate en la comunidad educativa y ha estado indisociablemente presente en toda propuesta sostenible de educación e-learning (y por extensión natural también de b-learning). El OAR se concibe como un paradigma de buenas prácticas en moderna pedagogía debido a muchas de las beneficiosas señas de identidad que definen a todo OAR correctamente elaborado y que han sido descritas amplia y profusamente en la literatura (Polsani, 2003). Las características inherentes a los objetos de aprendizaje reutilizables nos pueden servir de pauta para la elaboración de vídeos educativos ex novo y que transciendan de un uso ocasional y local (para alguna asignatura muy concreta) para convertirse en verdaderos instrumentos pedagógicos que compartir con otros docentes de materias afines. Es importante hacer notar en este punto la sutil (pero no vana) diferencia entre la utilización con propósitos educativos de un vídeo de carácter general (o incluso con intencionalidad pedagógica pero de libre elaboración) y la realización de vídeos específicamente educativos y sujetos a la filosofía de un OAR. Todos sabemos que existen organizaciones muy prestigiosas que elaboran multitud de magníficos vídeos sobre diferentes temas científicos y con carácter divulgativo (por ejemplo, National Geographic, BBC, etc.) y que pueden tener indudable utilidad pedagógica, pero que por su formato, derechos de propiedad, accesibilidad y otras caracteristicas, difícilmente encajarian en la categoría de objetos de aprendizaje reutilizables. Sin embargo, resulta natural concebir la realización de vídeos educativos elaborados dentro del marco conceptual que define un objeto de aprendizaje reutilizable, pues la unión de las modernas tecnologías audiovisuales y de las TIC, permiten producir excelentes materiales pedagógicos que satisfagan todas las caracteristicas analizadas con anterioridad. En efecto, mediante instrumentos de grabación con soporte digital junto con las potentes herramientas de edición no-lineales que ofrece actualmente el mercado de software y pudiendo alojar y compartir el producto realizado en la red, se da cumplida cuenta a los criterios de objeto visual de naturaleza digital, reutilizable, accesible, flexible y auto-contenido.

\section{El anAlizador de espectros: un caso DE APLICACIÓN DE VÍDEOS OAR}

Desde hace varios años los profesores del Grupo de Innovación Educativa en Sistemas de Telecomunicación (GIE-ST) de la Universidad Politécnica de Madrid, han percibido la necesidad de ir creando material educativo que pudiera ser utilizado por docentes y alumnos más allá de las fronteras de espacio y tiempo impuestas por una aula en la que se imparte una clase presencial convencional. Puesto que el grupo es multidisciplinar así también es el resultado del material que ha ido elaborando (radiocomunicaciones, antenas, comunicaciones ópticas, electrónica de comunicaciones) y el soporte, herramientas o tecnologías empleadas (Matlab, C++, 3d Studio Max, DVD y otras muchas. En algunos casos el material se ha elaborado con vistas a poder ser puesto a disposición de cualquier interesado, para lo cual se ha utilizado la popular plataforma de YouTube. En general, sin embargo, se ha adolecido de un criterio unificador a la hora de plantearse tanto el formato físico como la forma de utilización del material educativo a producir.

La reciente experiencia adquirida por el GIE-ST al trabajar conjuntamente con otros grupos de innovación educativa de la UPM, ha impulsado la adopción de criterios unificadores tanto en lo que se refiere a los medios de producción del material como a su ulterior uso. En esta línea se ha realizado (y se continúa aún) una experiencia de innovación educativa que se sustenta en cuatro etapas básicas 
(que pueden verse esquemáticamente representadas en la figura 2) generando un flujo cíclico de: búsqueda de material pedagógico (en) abierto en la red, análisis crítico de aplicabilidad, producción de material educativo específico, alojamiento en abierto en la red.

En lo que se refiere a la primera de las actividades llevadas a cabo, se ha realizado una búsqueda exhaustiva a través de Internet, de repositorios institucionales o privados (pero accesibles) y de sitios web (fundamentalmente universidades) que pudieran contener material educativo abierto y de calidad dentro de los ámbitos de trabajo o docente de los profesores que forman parte del GIE-ST. En esta labor han trabajado de forma cooperativa también alumnos de grado y becarios, al objeto de fomentar en ellos una capacidad de análisis crítico y selectivo del ingente material que puede encontrarse en la red.

Concluida la fase de minería de datos anterior, se ha realizado un proceso de análisis crítico del material encontrado, clasificándolo según diferentes parámetros (calidad, reutilizabilidad, accesibilidad, grado de adaptabilidad al nivel de las asignaturas regladas impartidas, etc.). La última acción dentro de esta etapa, ha sido la de búsqueda de un ítem que fuera común denominador dentro del abanico de asignaturas que los profesores del GIE-ST imparten y su disponibilidad dentro del material buscado, depurado y analizado. Como fruto de este proceso se llegó a la doble conclusión de que:

A. El Analizador de Espectros (AE) es quizás el instrumento de medida más utilizado dentro de los diferentes campos docentes de interés del GIEST, pues se emplea en prácticamente todas las bandas de frecuencias y para aplicaciones que van desde el análisis de una simple señal hasta el comportamiento de sistemas de telecomunicación complejos.

B. No se ha encontrado apenas material en abierto de calidad sobre AE. En cualquier caso, lo escaso localizado no cumple en modo alguno con los criterios propios de un OAR.

A la luz de las conclusiones precedentes se tomó la decisión de producir una biblioteca de vídeos sobre el funcionamiento y utilización de los analizadores de espectros siguiendo los condicionantes propios de un OAR y "subir- los" posteriormente a Internet para cumplir con los requisitos de total accesibilidad y fomentar su reutilización. En concreto se adoptaron los siguientes criterios (o buenas prácticas) como puntos de partida para la realización de cada uno de los vídeos:

1. Duración no superior a 3 minutos. Se considera que este tiempo constituye un buen compromiso que permite desarrollar una (o muy pocas) ideas principales en cada vídeo sin añadir contenido superfluo. Esta "píldora educativa" audiovisual debe producir adicionalmente un efecto colateral de incentivación a visionar el resto de vídeos de la biblioteca.

2. $\quad$ Nivel intermedio de contenido técnico. Se ha pretendido buscar un equilibrio entre amenidad de exposición y rigor científico en cada idea expuesta, al objeto de que pueda resultar útil al mayor público interesado posible. Un tratamiento superficial aportaría muy poco valor pedagógico y un desarrollo muy profundo podría producir un efecto de rechazo sobre el potencial usuario.

3. Potenciar los aspectos visuales frente a los formales. Esto quiere decir que las ideas a transmitir, aun teniendo un profundo soporte matemático, se expondrán mediante apoyatura de imágenes (tanto reales como animaciones) en tanto sea posible, introduciendo sólo la formulación matemática cuando sea imprescindible.

4. ${ }^{\circ}$ Utilización de locución mediante síntesis digital de voz. Hay dos razones básicas que justifican la adopción de este criterio. La primera es de naturaleza práctica y técnica, y estriba en que es inconmensurablemente más sencillo adaptar la duración del texto a la evolución de las imágenes que si se hiciese por un sistema analógico más convencional. Además, si fuera necesario resulta muy sencillo reeditar y modificar un fragmento de texto e incorporarlo de nuevo al vídeo de partida utilizando esta tecnología (se realiza en cuestión de escasos minutos), que realizar una grabación real frente a un micrófono (intentando mantener el mismo tono de voz, velocidad de lectura, etc.) y después incorporarlo otra vez con la duración exacta y en el punto preciso del vídeo matriz. Esta última opción requiere además mayor infraestructura 
y tiempo de edición. La segunda razón se basa en el intento de fomentar el grado de participación de los alumnos en la elaboración del material, haciendo que éstos aporten sus propios comentarios e ideas sobre las imágenes. Afortunadamente el estado del arte en la síntesis digital de voz ha permitido una locución con una aceptable expresividad y prácticamente nulo efecto "robotizado".

5. $\quad$ Difusión por medio de la plataforma YouTube ${ }^{\circledR}$. Se ha elegido este sitio web como la ubicación más propicia para difundir y compartir el material creado. Aunque se sopesaron otras alternativas (Moodle, página web del propio departamento, redes sociales, etc.) se estimó que tanto por la capacidad de alojamiento, calidad de imagen (hasta $1920 \times 1080$ pixeles máximo) y de audio (codificación AAC con dos canales a 44,1 KHz) ofrecidos, nivel mundial de difusión y capacidad de edición on-line (para generar enlaces dinámicos entre vídeos relacionados), resultaba el medio más idóneo para los objetivos buscados en la experiencia educativa.

\section{Resultados y futuros trabajos}

Como fruto de la iniciativa emprendida, el GIE-ST, ha producido hasta el momento cinco vídeos que versan sobre los

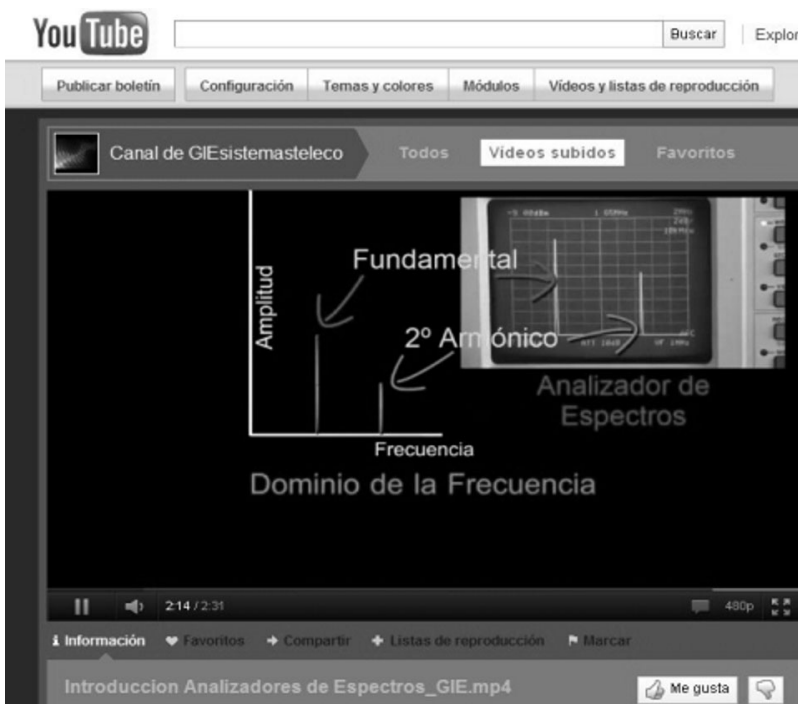

fundamentos y estructura de los analizadores de espectros, con una duración conjunta en torno a los 10 minutos y son accesibles a través del canal "GIEsistemasteleco" de YouTube. Una muestra visual del trabajo puede apreciarse en las dos capturas de pantalla mostradas en las figuras 3 y 4 . El proceso de producción continúa en la actualidad generando nuevos vídeos que se irán paulatinamente incorporando al corpus de materiales docentes ofrecidos a los alumnos. Se ha realizado un análisis de los resultados académicos obtenidos en las asignaturas de prácticas de laboratorio, de un grupo de alumnos que han utilizado los vídeos ya elaborados como material de apoyo y se han contrastado con las calificaciones de los restantes estudiantes que no han hecho uso de este material, verificándose una clara correlación entre la mejora en la adquisición de conocimientos objetivos y de habilidades de uso de instrumentación del primer conjunto de alumnos mencionado. Adicionalmente se ha sometido el material videográfico al juicio de los estudiantes con el objetivo de extraer conclusiones que sirvan de pautas para mejorar el proceso de producción de los nuevos vídeos planificados.

\section{AGRADECIMIENTOS}

Quisiéramos mostrar nuestra gratitud a Jon Artiaga por su colaboración y aportaciones, así como a Ana Giménez de los Galanes por cuidar de la inteligibilidad de esta comunicación.

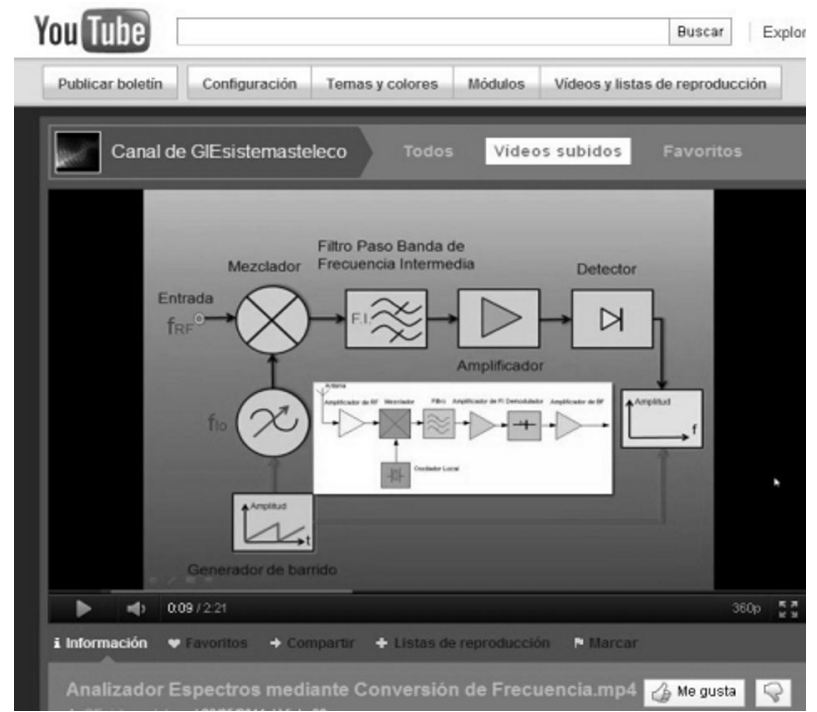

Figuras 3 y 4. Fotogramas de dos de los vídeos producidos. 
NOTA

1 También Ilamado Objeto Educativo Reutilizable (OER).

\section{BIBLIOGRAFÍA}

Bersin, J. (2004): The Blended Learning Book-Best Practices, Proven Methodologies and Lessons Learned, Editorial Pfeiffer, San Francisco.

Davoli, F.; Meyer, N.; Pugliese, R. y Zappatore, S. (2010): Remote Instrumentation and Virtual Laboratories-Service Architecture and Networking, Editorial Springer Science.

Duun, R. y Griggs, S. A. (2000): Practical Approaches to Using Learning Styles in Higher Education, Editorial Bergin \&t Garvey, Westpot.

Gruber, C. I.: El vídeo como recurso didáctico, Aula del Pedagogo. Disponible online en http://www.auladelpedagogo. com/2011/03/el-video-como-recursodidactico/

Polsani, P. R. (2003): "Use and Abude of Reusable Learning Objects", Journal of Digital Information, vol. 3, n. ${ }^{\circ}$ 4. Disponible on-line en http://www.info2. uqam.ca/ nkambou/DIC9340/.../ http__jodi.ecs.soton.ac.pdf.

Ynoue, Y. (2010): Cases on Online and Blended Learning Technologies in Higher Education-Concepts and Practices, Editorial Information Science Refrence, New York. 